\title{
IL POTERE DEL SOGNO: MUSICA E STRUTTURA NEL FILM EYES WIDE SHUT DI
}

\author{
Emanuele Ferrari \\ Universitá Bicocca, Milano
}





\section{Una struttura musicale}

La fonte di Eyes Wide Shut (USA, 1999) è il racconto Doppio Sogno di Arthur Schnitzler, scrittore attento alle profondità della psiche umana. La via scelta da Kubrick è però queIla seguita in molti altri film: utilizzare questa fonte letteraria come puro materiale da costruzione, dando vita ad un prodotto del tutto originale.

La prima parte del film ${ }^{102}$ è dominata dal caso: alla festa di Ziegler Bill è interrotto nel flirt con le modelle dalla chiamata del padrone di casa; più avanti, è strappato alla confessione di Alice dallo squillo del telefono, e così via. La seconda parte, per contro, è segnata dalla volontà di Bill, che decide di fare ricerche e tenta delle indagini, ripercorrendo i passi del giorno prima come un moderno, straniato Pollicino.

Ma proprio il caso produce, nella prima parte, una sorta di consequenzialità che cospira a far scivolare Bill verso la scena centrale dell'orgia, mentre l'esplorazione volontaria della seconda parte non frutta quasi altro che un ricalco disordinato di luoghi e situazioni già viste. I luoghi vengono ripercorsi in un ordine sparso, che ora appare -paradossalmente- casuale. II caso sembra dunque produrre una parvenza di consequenzialità, mentre la volontà cosciente approda al caos.

Questa struttura è debitrice di principi formali che vengono dalla musica. In primo luogo il film ha la forma di una canzone tripartita ABA', dove B è la scena dell'orgia, A tutto ciò che la precede e $A^{\prime}$ tutto ciò che segue. Inoltre, è parzialmente assimilabile ad un Rondò di luoghi e situazioni, in cui il Ritornello, cioè l'elemento che ritorna periodicamente, è la casa di Bill. Infine, lo schema del film richiama in parte le cosiddette forme ad arco, in cui la seconda parte del percorso è il rovescio della prima (del tipo ABACABA).

\section{Sogno, desiderio, visione}

L' effetto di questo lavoro sulla struttura è quello di mettere i personaggi in balia del montaggio. II protagonista si muove in uno spazio non euclideo e in un tempo non galileiano, percorrendo un labirinto di situazioni che immettono l'una nell'altra con logica incomprensibile. II vero protagonista del film non è Bill, bensì la galleria di luoghi, situazioni e personaggi che si presentano l'uno dopo l'altro con la logica bizzarra ma incontrovertibile dei sogni. Kubrick si appropria della dimensione onirica di Schnitzler facendo del sogno la logica della forma, caratterizzata tra l'altro dalla perdita di controllo, un tema caro al regista ${ }^{103}$. A questa perdita di controllo del soggetto fa riscontro un eccesso di senso delle cose. Tutto in Eyes Wide Shut irradia un senso, ora seducente ora maligno, mai completamente spiegabile, proprio come nei sogni. Le luci, i colori, le fisionomie dei luoghi e dei personaggi -lo sguardo astuto e diffidente da slavo di Milich, le luci che trasformano i manichini del suo negozio in creature fantastiche, il profilo da elegante avvoltoio del seduttore ungherese, e così via- sono tanto forti da fare di ogni personaggio e di ogni ambiente un universo chiuso, significativo ma del tutto inaccessibile. Mai come qui vale la definizione di Merleau-Ponty del corpo come "senso incarnato"104.

Trascinato nei gorghi di questo mondo saturo, Bill sembra a tratti soccombere, affogare nel senso, un senso lontanissimo da quello che pareva dare ordine alla sua vita e che scaturisce senza tregua dalle cose stesse, ancorate al carro fantastico del desiderio. Un desiderio che non è primariamente una dimensione dell'inconscio, come in Schnitzler, bensì la legge del visibile. La forza di Eyes Wide Shut non sta nell'esplorare l'interno dei personaggi. Al contrario, il potere del film sta nel mostrarci ciò che è fuori da quei personaggi, quel visibile enigmatico e pieno di senso che -grazie alla cinepresa di Kubrick- ci fa spalancare gli occhi. Ecco che lo sguardo del regista, a differenza di quello di Schnitzler, non si rivolge dietro e dentro i protagonisti, ma di fronte e intorno, dispiegando l'enigma del mondo in tutto il suo splendore. La dimensione saliente del film non è l'interiorità psichica, ma l'esteriorità del visibile, un visibile che non ha equivalenti concettuali e non cessa di irradiare senso sovrabbondante e sfuggente. C'è un legame profondo, allora, non solo tra sogno e desiderio, come vuole la psicoanalisi classica, ma anche tra desiderio e visione; il cinema di Kubrick, come la pittura secondo Merleau-Ponty, "porta alla sua estrema potenza un delirio che è la visione stessa, perché vedere è avere a distanza (...)"105.

\section{Tra splendore e squallore: musica e traffico}

Splendore del visibile non significa però trasfigurazione della realtà. Molte situazioni del film sono sordide, false o scontate. I rapporti umani sono stereotipati, le parole risapute. C'è un fondo anonimo dell'esistenza che è reso percepibile dai numerosi frammenti di traffico automobilistico che connettono o separano le scene. II traffico è l'opposto della storicità, della localizzazione e del radicamento: è pura situazione, non-luogo, casualità. Nel traffico l'identità personale si perde: non ci sono persone, ma automobili che si equivalgono. L'emblema del traffico è il suo stesso rumore, quello di uno sciame di veicoli immemori che rende i luoghi equivalenti e ne cancella l'identità sonora.

Buona parte della musica presente nel film ha la stessa funzione. Si tratta di ballabili, standard jazz, canzoni, "una ridda di motivi di danza e alcuni brani mediati dalla cultura pop" ${ }^{106}$, da When I Fall In Love a Strangers In The Night. L'intera scena della festa da Ziegler è trattata in questo modo. Se ne ha la riprova confrontando la musica "dal vivo", suonata dall'orchestra, con quella riprodotta durante la pausa dei musi- 
cisti, senza origine e senza soggetto: non cambia niente, è comunque musica di sfondo, immemore tappezzeria sonora. II suo effetto è quello di stendere una patina di sentimento anonimo e risaputo sulle cose; proprio come il rumore del traffico, questo uso della musica, anziché caratterizzare le situazioni rendendole uniche e inconfondibili, crea un' impressione di generico déjà $v u$, contribuisce a indebolire l'identità delle cose e, anche più del traffico, trasforma luci e ombre sullo schermo in immagini di repertorio. Le storie diventano risapute, $\mathrm{i}$ dialoghi banali, volti e ambienti sbiadiscono leggermente. E' come se la musica stingesse le immagini, rendendole meno individuali e più impersonali. In quello straordinario "esercizio di stile" che è Eyes Wide Shut, la musica contribuisce al sofisticato gioco tra splendore e squallore, in una singolare equivalenza di funzioni con il rumore del traffico: il traffico è la musica d'ambiente degli esterni, mentre la musica è il rumore del traffico degli interni. L'effetto, nei due casi, è lo stesso.

\section{La verità dell'estraneo: i monologhi}

Diverso è il caso dei brani musicali di Jocelyn Pook, Naval Officere The Dream, che accompagnano i due monologhi di Alice. Poiché le due scene sono simmetriche, ci limiteremo a quella in cui Alice racconta al marito il suo tradimento mentale. E' questo il primo momento narrativo del film, uno dei pochi in cui le parole sono fondamentali. Apparentemente è l'ora della verità, in cui Alice mette a nudo il suo animo. In realtà, la scena è un monumento all'estraneità: mai come qui si tocca con mano quanto l'universo degli altri sia lontano dal nostro. La voce della moglie, che fa da filo conduttore alla scena, si trasforma gradualmente per Bill da spettacolo famigliare in segno dell'alterità. II momento della verità non è quello in cui si perviene ad un'essenza condivisa delle cose, ma quello in cui balena folgorante l'inaccessibilità dell'altro. In totale coerenza con gli assunti del film, Kubrick dà il massimo risalto a questo scena, trasformando le immagini in un monumento alla bellezza di Nicole Kidman, qui divenuta enigmatica, e il racconto in un apologo, grazie alla musica che lo rende immobile come una parabola evangelica. II brano musicale non segue il racconto nei suoi soprassalti emotivi e si mantiene distante, lontano. L'assenza di movimento dà una ieratica dignità alla scena: la musica è idealmente lo spazio vuoto del tempio costruito intorno al monologo della dea, emanazione del tempo e della situazione. E' la dimensione dello sgomento, della lontananza, della presenza-assenza di un senso che possiamo intuire ma non toccare. II suono non riempie la scena; è evocativo ma remoto. Ciò impedisce allo spettatore di identificarsi totalmente con il personaggio e di farsi risucchiare dalla situazione, costringendolo ancora una volta, anziché a fare tutt'uno con le immagini, a guardarle.

\section{Eros e ambivalenza}

Da questo sfondo ora anonimo, ora informe, emergono alcune scene in cui la musica diviene figura in primo piano, imponendosi all'attenzione dello spettatore. Scegliamo quella in cui, dopo la festa, Alice è nuda davanti allo specchio e Bill le si avvicina dando avvio ai preliminari dell'amplesso.

Lo stacco tra la scena precedente -la festa da Ziegler- e questa, è uno stacco sonoro, prima che visivo. II passaggio dall'anonimo sottofondo musicale dei saloni al prepotente attacco del basso di Baby Did a Bad Bad Thing di Chris Isaak è assai più crudo ed efficace del mutamento nelle immagini. Scena muta, come si conviene al manifestarsi dell'eros, in cui Kubrick, nel dar voce alla musica, non rinuncia ai consueti giochi di sfasamento e ambiguità. La semantica della musica, per una volta, sembrerebbe chiara. Paragonato ai tenui acquerelli della festa, questo brano fa l'effetto di un denso colore ad olio, così come l'eros trasgressivo ma velleitario del seduttore ungherese impallidisce di fronte a quello concreto e consumato della coppia. "Qui si fa davvero sul serio", sembra dire un tale contrasto musicale; ma, come spesso in Kubrick, le immagini divergono. Alice accetta l'erotico cimento di Bill, ma volge gli occhi alla cinepresa con l'espressione sfuggente che è divenuta l'immagine simbolo del film. Se la musica sembra "parlare chiaro" a favore dell'eros "coniugale" di Bill, le immagini mostrano un'Alice ambivalente e lontana dall'aver dimenticato le avances trasgressive ricevute alla festa.

\section{Dal centro della terra}

Al vertice della piramide che stiamo delineando, caratterizzata da una crescente collocazione in primo piano della musica, sta la lunga sequenza dell'orgia, punto culminante del film e spartiacque formale. Sembra di entrare in un sogno sconvolgente, ma l' effetto è raggiunto senza ingredienti convenzionali come luce irreale, nebbia, o sfumato. Al contrario, tutto è incredibilmente nitido. La musica è il motore della scena, che non a caso rientra in una categoria, quella delle "scene rituali", che Frits Noske ha studiato nel teatro d'opera ${ }^{107}$. II brano scelto da Kubrick, Masked Ball di Jocelyn Pook, è senz'altro efficace di per sé, ma a farne deflagrare la potenza espressiva è ancora una volta l'abbinamento con le immagini. Nel contesto ieratico delle maschere, dei mantelli e dei corpi nudi, la musica fa un'enorme impressione: il canto sinistro e gorgogliante sembra provenire da un cratere spalancato fino al centro della terra, e lo spettatore è portato inconsciamente a cercarne la fonte nell'officiante mascherato, 0 in Nick, che suona l'accompagnamento sulle tastiere elettroniche. Entrambe le ipotesi si rivelano false quando, con sconcerto, si scopre che Nick sta suonando a bocca chiusa, e soprattutto che la melodia si sdoppia e le voci diventano due. L'effetto è straordinario: l'intera scena è immersa nella musica, che sembra erompere dai corpi e ad essi ritornare, tanto è imbevuta di violenta fisicità rituale, eppure non si è in grado di 
identificarne la provenienza. La dimensione onirica tocca qui un climax difficilmente superabile. La musica detta il ritmo, i gesti e il modo di muoversi di tutti i personaggi, e ad essa sembrano obbedire persino le lente movenze rotatorie della cinepresa, ipnotizzata dalla musica come un serpente da un incantatore. Le maschere non fanno che accentuare lo stupore onirico dello spettatore, deformando i lineamenti in una smorfia eterna e distorcendo le voci che ancora non si odono. II canto spaventoso e arcano che risuona nella sala come in una caverna sembra, a questo punto, voce di tutti e di nessuno: l'impressione è quella di sognare ad occhi aperti.

\section{II valzer delle occasioni perdute}

Resta un brano con una storia a sé, il valzer dalla Jazz Suite di Šostakovič che compare nei titoli di testa e di chiusura. E' un pezzo intenso, malinconico, decadente, che sembra promettere al pubblico una grande storia romantica e dà l'idea che la vita stia per scorrerci davanti in tutta la pienezza dei suoi significati. Ma questa promessa, come abbiamo visto, non viene affatto mantenuta.

II valzer ritorna per l'ultima volta sui titoli di coda, con la più sottile delle ironie. Se il film fosse una commedia sentimentale americana per famiglie, questa musica accompagnerebbe la rievocazione finale dei personaggi, colti uno ad uno nelle foto dei momenti più intensi. Ma qui non abbiamo visto alcun film del genere. In Eyes Wide Shut la pienezza del senso visibile convive con un vuoto di senso intelligibile; il protagonista ha perso il controllo sulla sua vita, ha corso per caso gravi pericoli e solo per caso ne è uscito. L'unica acquisizione è la radicale ambiguità delle cose, l'eccedenza di un senso che è fascinoso e sovrabbondante per l'occhio ma inaccessibile ed enigmatico per la ragione. La malinconia non proviene allora dal commiato che corona una ricca esperienza, ma dalla sensazione che questa musica parli di una storia che non c'è stata, di una vita che non abbiamo vissuto, di occasioni perdute che Bill, e lo spettatore con lui, hanno solo sfiorato. Questa musica evoca un mondo di destini vissuti intensamente e padroneggiati, ma lo fa paradossalmente a conclusione di una sequela di atti mancati. E' il commiato da un film che non abbiamo visto, intriso di nostalgia per una pienezza del vivere che ci è stata sottratta...

$\mathrm{E}$ torna in mente 0 . Wilde quando dice che la musica ci fa sognare un passato personale che non abbiamo mai vissuto davvero ${ }^{108}$.

\section{Notas}

102 Per ragioni di spazio, consideriamo nota la trama al lettore.

103 Ne parla diffusamente, ad esempio, R. Eugenii in Invito al cinema di Kubrick, Milano, Mursia, 1995, nei paragrafi del terzo capitolo dedicati alla "crisi del controllo".

104 Merleau-Ponty, M. Phénoménologie de la perception, Gallimard, Paris, 1945; tr. it. di A. Bonomi, Fenomenologia della percezione, Bompiani, Milano, 2003, p. 234.

105 Merleau-Ponty, M. L'CEil et l'Esprit, Gallimard, Paris, 1964; tr. it. di A. Sordini, L'occhio e lo spirito, SE, Milano, 1989, p. 23.

106 Bassetti, S. La musica secondo Kubrick, Lindau, Torino, 2002, p. 166.

107 Noske, F. The Signifier and the Signified. Studies in the Operas of Mozart and Verdi, Oxford, Oxford University Press, 1977; tr. it. di L. Minardi, Dentro l'opera. Struttura e figura nei drammi musicali di Mozart e Verdi, Marsilio, Venezia, 1993.

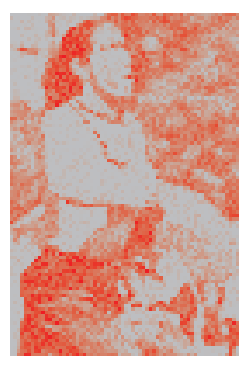

Emanuele Ferrari: E' ricercatore di musicologia e storia della musica all'Università MilanoBicocca presso la facoltà di Scienze della Formazione, dove insegna Musica e didattica della musica ed è coordinatore dei laboratori musicali. e-mail: ferrari.mus@libero.it emanuele.ferrari@unimib.it 